\title{
Ambiguity Tolerance and Prospective Specialty Choice Among Third-Year Medical Students
}

Oksana Babenko, PhD | Delane Linkiewich, BA | Kalee Lodewyk | Ann Lee, MD, MEd

PRiMER. 2021;5:2.

Published: 1/8/2021 | DOI: 10.22454/PRiMER.2021.453158

\section{Abstract}

Introduction: Poor tolerance of ambiguity is consequential in clinical practice, and has been linked to avoidance of family medicine, in which there is inherently more ambiguity. This study aimed to investigate the relationship between tolerance of ambiguity and prospective specialty choice of medical students in their third year of medical school. This stage of medical training is of particular importance as students develop clinical reasoning skills and encounter clinical ambiguity.

Methods: This was a cross-sectional study using an online survey. Sixty-one third-year medical students (62\% response rate) from a large Canadian university completed the survey with a validated measure of ambiguity tolerance (the 29-item Tolerance of Ambiguity in Medical Students and Doctors scale) and their top three specialty choices. Specialty choices were subsequently grouped into two categories: family medicine (FM) and non-family medicine (non-FM) specialties.

Results: There was no significant mean difference in tolerance of ambiguity between students who reported interest in FM and students interested in non-FM specialties. Similarly, we observed no significant difference in tolerance of ambiguity between female and male students. Older students reported higher levels of ambiguity tolerance. Older students were also more likely to report FM as one of their top three specialty choices.

Conclusion: Qualitative studies are needed to explore possible reasons for the observed results, including the effects of digital information resources and clinical decision-making tools on medical students' ambiguity tolerance. Medical educators should be aware that some students may require explicit training in how to respond to ambiguity.

\section{Introduction}

Poor tolerance of ambiguity is consequential in clinical practice (eg, increased diagnostic testing, unnecessary referrals). ${ }^{1}$ Some studies suggest that it may also be linked to lower preference for family medicine. ${ }^{2,3}$

Family medicine may be more attractive to learners if explicit training to respond to ambiguity adaptively is implemented. ${ }^{1}$ In Canada and the United States, medical students in their third year of medical training develop clinical reasoning skills through clinical rotations. This is also the time when students select electives and begin choosing specialties. As such, we sought to investigate the level of ambiguity tolerance among third-year 
medical students and its association with prospective specialty choice.

\section{Methods}

This was a survey study of a third-year medical student cohort at the University of Alberta, Canada, in the 2019-2020 academic year. We obtained ethics approval from the institutional research ethics board prior to data collection. Sixty-one third-year medical students completed the questionnaire (62\% response rate). Participants' demographic information is shown in Table 1.

We used the 29-item Tolerance of Ambiguity in Medical Students and Doctors (TAMSAD) scale 4 in the study. Although it was developed in the United Kingdom, we chose the TAMSAD scale based on validity evidence for its use with medical students and junior doctors. ${ }^{4}$ Our intention is to follow up with the study participants for changes, if any, in ambiguity tolerance and their eventual specialty choice. Using a 5-point Likert-type scale (1strongly disagree; 5-strongly agree), students were asked to indicate the level of agreement with each statement. After averaging student responses to 29 items, we linearly transformed the resultant scores to the 0-100 scale using the formula suggested by the scale developers where "transformed score $=25^{\star}$ (average score-1)." ${ }^{4}$ Higher transformed scores were indicative of greater tolerance of ambiguity. Students were also asked to list their preferred top three specialty choices. For the purpose of this study, we grouped students' preferred top three specialty choices into two categories, namely, family medicine ([FM], which in Canada is synonymous with primary care) and non-family medicine (non-FM) specialties.

With SPSS 26.0 software, we used descriptive statistics to examine the level of agreement with each TAMSAD item by the students and their overall level of ambiguity tolerance. We used Spearman's correlation and $\chi^{2}$ tests to examine bivariate associations. We used independent samples $t$ test to test for mean differences in students' ambiguity tolerance based on gender and preferred specialty choice. We computed effect sizes of mean differences using Hedges' $\mathrm{g}$. We used an a level of 0.05 and Bonferroni correction for multiple comparisons in significance testing.

\section{Results}

The mean TAMSAD score was 60.80 (SD=7.85; min-max=42.24-83.62), which is five points higher than the mean score reported by the TAMSAD scale developers for the third-year medical students in their study (Hedges' $g=0.79) .{ }^{4}$

Of those participants who reported their preferred specialty, 50.8\% $(n=30)$ indicated FM among top three specialty choices, and $49.2 \%(n=29)$ indicated a preference for non-FM specialties. There was no significant mean difference in ambiguity tolerance between students who preferred FM and students who preferred nonFM specialties as their top three specialty choices $(t=0.657, \mathrm{df}=57, P=.514$; Table 1$)$. However, older students (25-29 years) were more likely than younger students (20-24 years) to report FM as one of their top three specialty choices $(63.3 \%$ vs $38.5 \%$, respectively; $P=.031)$.

There was no significant mean difference in ambiguity tolerance between female and male students $(t=0.363$, $\mathrm{df}=58, P=.718$; Table 1 ), which is consistent with findings reported by the TAMSAD scale developers. ${ }^{4}$ The association between students' age and ambiguity tolerance was positive and of moderate size (Spearman's $\rho=0.31, P=.016$ ), indicating a possible maturation effect in students' ability to tolerate ambiguity.

\section{Conclusions}

On average, we observed higher ambiguity tolerance among third-year medical students than that reported by 
the TAMSAD scale developers in $2015 .{ }^{4}$ While it may be due to inherent differences in populations and/or educational frameworks, recent literature suggests there may be other factors at play. One such factor is that current medical students are predominantly from a digitally native generation. ${ }^{5}$ They are more likely to use online resources to access information to support their clinical decision making because of the familiarity and availability of such resources. ${ }^{6}$ Qualitative studies, employing focus groups and interviews with medical students, are needed to explore the reasons for higher ambiguity tolerance, including the possible role of digital resources and tools in current students' perceptions of clinical ambiguity.

Despite an average higher ambiguity tolerance, the observed variation in individual students' TAMSAD scores in this study as well as in the scale development study ${ }^{4}$ supports the suggestion that medical educators should encourage ambiguity tolerance earlier in training, such as in preclinical years. ${ }^{7}$ Incorporating multiple teaching strategies (eg, using grey cases; acknowledging where ambiguity is present) is needed to facilitate students' tolerance of ambiguity. ${ }^{7}$

In contrast to literature that suggests a potential link between ambiguity tolerance and specialty choice, we observed comparable ambiguity tolerance in students who indicated preference for FM and students who indicated preference for non-FM specialties. Recent literature offers some possibilities that should be explored in relation to this result. Until recently, FM has been regarded as a specialty with inherently more ambiguity than other specialties. ${ }^{8,9}$ While every specialty has some degree of ambiguity, the ambiguities that exist in other specialties occur in FM. In recent years, increased availability of clinical guidelines, ${ }^{10}$ tools for practice, ${ }^{11}$ medical search engines, ${ }^{12}$ and medical applications for smartphones and tablets ${ }^{13}$ have enabled physicians to better respond to ambiguity in clinical decision making. It is plausible that current medical students undergoing clinical rotations in FM clinics are witnessing positive effects of such tools in reducing clinical ambiguity and as such, ambiguity tolerance may no longer be an important factor when choosing FM as a specialty. Future studies are needed to test this speculation.

This study has limitations. Although our medical program is similar to those in other North American medical schools, data collection took place at one Canadian school, limiting generalizability of the results. This exploratory study suggests that a multicentered study is needed. Additionally, given the focus on third-year medical students, the sample size in this study was limited. However, the response rate was moderately high and consistent with response rates reported in survey-based studies in health professions education. ${ }^{14}$ Despite the size, the sample was balanced in terms of students' gender, and $50 \%$ of students in this study indicated interest in FM, which is consistent with the Canadian Resident Matching Service statistics, ${ }^{15}$ mitigating potential nonresponse bias. Next, although we did not observe a relationship between ambiguity tolerance and specialty choice using a survey approach, posing clinically based questions may provide a better understanding of whether students may avoid certain specialties, including other generalist careers such as internal medicine, paediatrics, and general surgery, due to poor ambiguity tolerance. While in Canada FM is synonymous with primary care, examining the relationship between ambiguity tolerance and specialty choice of primary care vs non-primary care should be considered in other countries where primary care may include other specialties such as internal medicine and pediatrics. Finally, interest in family medicine was defined as listing it as one of the top three specialties, which is a broad definition that could have influenced the findings.

Despite these limitations, this study replicated some of the results reported in the TAMSAD scale development study ${ }^{4}$ in the Canadian context. While ambiguity tolerance may not be playing a significant role in specialty choice, the observed higher level of ambiguity tolerance among this contemporary sample of students warrants further research. At the same time, the variation in ambiguity tolerance supports the need for medical educators to help learners develop adaptive responses to clinical ambiguity earlier in their training, given the potential for such responses to combat unnecessary diagnostic testing and referrals. ${ }^{1}$ 


\section{Tables and Figures}

Table 1: Participant Characteristics and Mean Scores (Standard Deviations [SD]; Min-Max Scores) on the TAMSAD Scale $(n=61)^{a}$

\begin{tabular}{|l|c|c|}
\hline \multicolumn{1}{|c|}{ Characteristics } & $\%(\mathrm{n})$ & Mean (SD; Min-Max) \\
\hline $\begin{array}{l}\text { Gender }(\mathrm{n}=60) \\
\text { Female }\end{array}$ & $50.0(30)$ & $61.12(8.56 ; 42.24-83.62)$ \\
Male & $50.0(30)$ & $60.37(7.32 ; 43.97-73.28)$ \\
\hline Age in Years $(\mathrm{n}=60)$ & $45.0(27)$ & \\
$20-24$ & $50.0(30)$ & $58.24(8.76 ; 42.24-83.62)$ \\
$25-29$ & $5.0(3)$ & $62.87(6.80 ; 45.69-75.86)$ \\
$30-34$ & $50.8(30)$ & $62.07(4.56 ; 56.90-65.52)$ \\
\hline Specialty Choice $(\mathrm{n}=59)$ & $49.2(29)$ & $59.91(7.91 ; 42.24-75.86)$ \\
Family medicine & $61.23(7.89 ; 45.69-83.62)$ \\
\hline Non-family medicine &
\end{tabular}

a One participant chose not to indicate gender and age and two participants did not indicate their preferred specialty choice.

\section{Acknowledgments}

Financial Support: This work was supported by research grants from the Social Sciences and Humanities Research Council (SSHRC) of Canada (Grant \# 430-2016-00267), the Northern Alberta Academic Family Physicians Fund (NAAFP), and the Health Professions Education Summer Studentship Fund at the University of Alberta, Canada.

Presentation: Preliminary findings were presented at the Family Medicine Summit of the Alberta College of Family Physicians, March 6, 2020, in Banff, Alberta, Canada.

\section{Corresponding Author}

Oksana Babenko, PhD

Department of Family Medicine, 6-10 University Terrace, University of Alberta, Edmonton, Alberta, Canada T6G 2T4. 1-780-248-1729. Fax: 1-780-492-2593.

oksana.babenko@ualberta.ca

\section{Author Affiliations}

Oksana Babenko, PhD - Department of Family Medicine, University of Alberta, Edmonton, Alberta, Canada Delane Linkiewich, BA - Department of Family Medicine, University of Alberta, Edmonton, Alberta, Canada Kalee Lodewyk - Department of Family Medicine, University of Alberta, Edmonton, Alberta, Canada Ann Lee, MD, MEd - Department of Family Medicine, University of Alberta, Edmonton, Alberta, Canada

\section{References}

1. Kim K, Lee Y-M. Understanding uncertainty in medicine: concepts and implications in medical education. Korean J Med Educ. 2018;30(3):181-188. doi:10.3946/kjme.2018.92

2. Nevalainen M, Kuikka L, Sjoberg L, Eriksson J, Pitkala K. Tolerance of uncertainty and fears of making mistakes among fifth-year medical students. Fam Med. 2012;44(4):240-246.

3. Caulfield M, Andolsek K, Grbic D, Roskovensky L. Ambiguity tolerance of students matriculating to U.S. medical schools. Acad Med. 2014;89(11):1526-1532. doi:10.1097/ACM.0000000000000485

4. Hancock J, Roberts M, Monrouxe L, Mattick K. Medical student and junior doctors' tolerance of ambiguity: development of a new scale. Adv Health Sci Educ Theory Pract. 2015;20(1):113-130. 


\section{doi:10.1007/s10459-014-9510-z}

5. Stan VA, Correa R, Deslauriers JR, Faynboym S, Shah T, Widge AS; AMA-RFS Task Force on Resident/Fellow Physician Satisfaction. Support, technology and mental health: correlates of trainee workplace satisfaction. Perspect Med Educ. 2020;9(1):31-40. doi:10.1007/s40037-019-00555-2

6. Wynter L, Burgess A, Kalman E, Heron JE, Bleasel J. Medical students: what educational resources are they using? BMC Med Educ. 2019;19(1):36. doi:10.1186/s12909-019-1462-9

7. Stephens GC, Rees CE, Lazarus MD. Exploring the impact of education on preclinical medical students' tolerance of uncertainty: a qualitative longitudinal study. Adv Health Sci Educ Theory Pract. 2020. doi:10.1007/s10459-020-09971-0

8. Katerndahl D, Wood R, Jaén CR. Family medicine outpatient encounters are more complex than those of cardiology and psychiatry. J Am Board Fam Med. 2011;24(1):6-15. doi:10.3122/jabfm.2011.01.100057

9. Alam R, Cheraghi-Sohi S, Panagioti M, Esmail A, Campbell S, Panagopoulou E. Managing diagnostic uncertainty in primary care: a systematic critical review. BMC Fam Pract. 2017;18(1):79. doi:10.1186/s12875-017-0650-0

10. Dickinson JA, Bell NR, Grad R, Singh H, Groulx S, Szafran O. Choosing guidelines to use in your practice. Can Fam Physician. 2018;64(5):357-362.

11. Allan GM, Ivers N, Pimlott N. Introducing Tools for Practice. Can Fam Physician. 2010;56:556.

12. Labrecque $M$, Ratté $S$, Frémont $P$, et al. Decision making in family medicine: randomized trial of the effects of the InfoClinique and Trip database search engines. Can Fam Physician. 2013;59(10):1084-1094.

13. Lippman $\mathrm{H}$. How apps are changing family medicine. J Fam Pract. 2013;62(7):362-367.

14. Phillips AW, Friedman BT, Utrankar A, Ta AQ, Reddy ST, Durning SJ. Surveys of health professions trainees: Prevalence, response rates, and predictive factors to guide researchers. Acad Med. 2017;92(2):222-228. doi:10.1097/ACM.0000000000001334

15. 2019 R-1 match data snapshot. The Canadian Resident Matching Service. https://www.carms.ca /pdfs/2019-R-1-2-data-snapshot.pdf. Accessed September 9, 2020.

Copyright $\odot 2021$ by the Society of Teachers of Family Medicine 